\title{
INVOLUTIONS FIXING PRODUCTS OF CIRCLES
}

\author{
R. E. STONG \\ (Communicated by Thomas G. Goodwillie) \\ In memory of $E$. E. Floyd
}

\begin{abstract}
This paper determines the possible equivariant bordism classes of involutions having fixed set a union of products of circles.
\end{abstract}

\section{INTRODUCTION}

Consider the involution on the projective plane $R P^{2}$ defined by

$$
T\left(\left[x_{0}, x_{1}, x_{2}\right]\right)=\left[-x_{0}, x_{1}, x_{2}\right] \text {. }
$$

The fixed point set of this involution consists of a point, $[1,0,0]$, with trivial normal bundle and a circle, $S^{1}$ or $R P^{1}$, given by the points with $x_{0}=0$, with normal bundle the nontrivial line bundle $\xi$ over $R P^{1}$.

Forming the product of $m$-copies of this example, one obtains an involution on $\left(R P^{2}\right)^{m}=R P^{2} \times \cdots \times R P^{2}$ given by $T \times \cdots \times T$, for which the fixed point set is the union of $\left(\begin{array}{c}m \\ k\end{array}\right)$ copies of $\left(R P^{1}\right)^{k}$ with normal bundle

$$
\xi_{1} \oplus \xi_{2} \oplus \cdots \oplus \xi_{k} \oplus(2 m-2 k) \rightarrow R P^{1} \times \cdots \times R P^{1}
$$

for $0 \leq k \leq m$. Here $\left(\begin{array}{c}m \\ k\end{array}\right)$ is the binomial coefficient, $\xi_{i}$ is the line bundle over the $i$ th factor, and $\left(R P^{1}\right)^{0}$ is interpreted as being a point.

In their book [2] Conner and Floyd proved that, up to bordism, $\left(R P^{2}, T\right)$ is the only involution with fixed set the union of a point and a circle. (See [2, (27.6)].) The purpose of this note is to establish the generalization:

Theorem. If $\left(M^{n}, T\right)$ is an involution having fixed point set a union of copies of $\left(R P^{1}\right)^{k}$, with $0 \leq k \leq n$, then either $\left(M^{n}, T\right)$ bounds or $n=2 m$ and $\left(M^{n}, T\right)$ is equivariantly cobordant to the involution $\left(\left(R P^{2}\right)^{m}, T \times \cdots \times T\right)$.

The author is indebted to the National Science Foundation for financial support during this work.

\section{THE PROOF}

From [2, (28.1)], one has an exact sequence

$$
0 \rightarrow \mathscr{N}_{n}^{Z_{2}} \stackrel{F}{\rightarrow} \bigoplus_{j=0}^{n} \mathscr{N}_{n-j}\left(B O_{j}\right) \stackrel{\partial}{\rightarrow} \mathscr{N}_{n-1}\left(R P^{\infty}\right) \rightarrow 0,
$$

Received by the editors March 16, 1992.

1991 Mathematics Subject Classification. Primary 57R85. 
which shows that the cobordism class of an involution is determined by the bordism class of its fixed set and normal bundle. To study involutions fixing unions of products of $R P^{1}$ 's one needs

Lemma. If $\eta^{j} \rightarrow\left(R P^{1}\right)^{k}$ is a j-plane bundle over the $k$-fold product which is nonbounding (in $\mathscr{N}_{k}\left(B O_{j}\right)$ ), then $j \geq k$ and $\left(\left(R P^{1}\right)^{k}, \eta^{j}\right)$ is bordant to the bundle

$$
\xi_{1} \oplus \cdots \oplus \xi_{k} \oplus(j-k) \rightarrow\left(R P^{1}\right)^{k}
$$

Proof. The bordism class of a bundle is determined by its Stiefel-Whitney numbers, and since $\left(R P^{1}\right)^{k}$ is parallelizable, the only Stiefel-Whitney numbers that can possibly be nonzero are those of the form

$$
w_{i_{1}} w_{i_{2}} \cdots w_{i_{r}}\left[\left(R P^{1}\right)^{k}\right]
$$

where $w_{i}=w_{i}(\eta)$ and $i_{1} \leq i_{2} \leq \cdots \leq i_{r}, i_{1}+\cdots+i_{r}=k$. Further, if $x \in H^{*}\left(\left(R P^{1}\right)^{k} ; Z_{2}\right)$, then $x^{2}=0$, and one may suppose $i_{1}<i_{2}<\cdots<i_{r}$. From Wu's theorem [3]

$$
S q^{s} w_{t}=\sum_{u=0}^{s}\left(\begin{array}{c}
t-s-1+u \\
u
\end{array}\right) w_{s-u} w_{t+u}
$$

and triviality of the action of the Steenrod algebra in $H^{*}\left(\left(R P^{1}\right)^{k} ; Z_{2}\right)$, one has

$$
0=S q^{2^{a}} w_{2^{a+1} b}=w_{2^{a+1} b} w_{2^{a}}+w_{2^{a+1} b+2^{a}} .
$$

Thus, the only Stiefel-Whitney numbers $w_{i_{1}} \cdots w_{i_{r}}\left[\left(R P^{1}\right)^{k}\right]$ with $i_{1}+\cdots+i_{r}=k$ which can be nonzero are those in which the $i$ 's have no common powers of 2 in their dyadic expansion, and these are nonzero if and only if $w_{k}\left[\left(R P^{1}\right)^{k}\right]$ is nonzero. If $\left(\left(R P^{1}\right)^{k}, \eta^{j}\right)$ is nonbounding, $w_{k}\left(\eta^{j}\right) \neq 0$, so $j \geq k$ and $\eta$ is bordant to $\xi_{1} \oplus \cdots \oplus \xi_{k} \oplus(j-k)$, which also has $w_{k}$ nonzero.

The proof of the theorem is now a very easy inductive argument. One considers a class

$$
\alpha=\left(\left(R P^{1}\right)^{k_{1}}, \eta^{n-k_{1}}\right) \cup \cdots \cup\left(\left(R P^{1}\right)^{k_{r}}, \eta^{n-k_{r}}\right)
$$

in $\sum_{k=0}^{n} \mathscr{N}_{k}\left(B O_{n-k}\right)$, where $k_{1}<k_{2}<\cdots<k_{r}$ and each bundle $\left(\left(R P^{1}\right)^{k}, \eta^{n-k}\right)$ is nonbounding. One can suppose $\eta^{n-k}=\xi_{1} \oplus \cdots \oplus \xi_{k} \oplus(n-2 k)$ with no loss, and hence $n \geq 2 k_{r}$.

The hypothesis for the induction on $k_{r}$ is that $\alpha$ is the fixed data of an involution $\left(M^{n}, T\right)$ if and only if $n=2 k_{r}$ and the $k_{i}$ occurring are precisely those for which $\left(\begin{array}{c}k_{r} \\ k_{i}\end{array}\right)$ is nonzero $\bmod 2$.

The case $k_{r}=0$ is trivial. One is asking that a point with trivial $n$-plane bundle be the fixed set of an involution $\left(M^{n}, T\right)$. This can only happen for $n=0$ with the trivial involution on a point, i.e., $\left(\left(R P^{2}\right)^{m}, T \times \cdots \times T\right)$ with $m=0$. (See [2, remark following (25.1)].) Now consider the class $\alpha$ and suppose $\alpha$ is the fixed set of an involution $\left(M^{n}, T\right)$. From [2, (26.4)], one has a commutative diagram

$$
\begin{array}{ccc}
\bigoplus_{k=0}^{n} \mathcal{N}_{k}\left(B O_{n-k}\right) & \multicolumn{2}{l}{\mathscr{N}_{n-1}\left(R P^{\infty}\right)} \\
\uparrow \bigoplus 1 & \downarrow \Delta \\
\bigoplus_{k=0}^{n-1} \mathcal{N}_{k}\left(B O_{n-1-k}\right) \stackrel{\partial}{\longrightarrow} \mathscr{N}_{n-2}\left(R P^{\infty}\right)
\end{array}
$$


where $\oplus 1$ adds a trivial line bundle and $\Delta$ is the Smith homomorphism.

Clearly, if $n>2 k_{r}$, then one has $\alpha=(\bigoplus 1)^{n-2 k_{r}} \alpha^{\prime}$, where

$$
\alpha^{\prime}=\left(\left(R P^{1}\right)^{k_{1}}, \eta^{2 k_{r}-k_{1}}\right) \cup \cdots \cup\left(\left(R P^{1}\right)^{k_{r}}, \eta^{k_{r}}\right)
$$

has the same sequence $k_{1}<\cdots<k_{r}$ as does $\alpha$. Then

$$
\partial \alpha^{\prime}=\Delta^{n-2 k_{r}} \partial(\bigoplus 1)^{n-2 k_{r}} \alpha^{\prime}=\Delta^{n-2 k_{r}} \partial \alpha=\Delta^{n-2 k_{r}} 0=0,
$$

so $\alpha^{\prime}$ is the fixed set of an involution on a manifold of dimension $2 k_{r}$. If $n=2 k_{r}, \alpha^{\prime}=\alpha$ and nothing has been done so far. Say $\alpha^{\prime}=F\left(N^{2 k_{r}}, S\right)$.

Now consider $\beta=F\left(\left(N^{2 k_{r}}, S\right) \cup\left(\left(R P^{2}\right)^{k_{r}}, T \times \cdots \times T\right)\right)$. This is the fixed data of an involution with

$$
\beta=\left(\left(R P^{1}\right)^{j_{1}}, \eta^{2 k_{r}-j_{1}}\right) \cup \cdots \cup\left(\left(R P^{1}\right)^{j_{s}}, \eta^{2 k_{r}-j_{s}}\right)
$$

having $j_{s}<k_{r}$ since the dimension $k_{r}$ components of the fixed sets in $\left(R P^{2}\right)^{k_{r}}$ and $N^{2 k_{r}}$ cancelled out. By the inductive hypothesis, this can only happen if $\beta=0$, for the dimension of the involution exceeds $2 j_{s}$.

Thus $\alpha^{\prime}=F\left(\left(R P^{2}\right)^{k_{r}}, T \times \cdots \times T\right)$ is the fixed set of the standard involution, or $k_{i}$ occurs precisely when $\left(\begin{array}{c}k_{r} \\ k_{i}\end{array}\right)$ is odd.

Now assume $n>2 k_{r}$. Then

$$
\partial(\bigoplus 1) \alpha^{\prime}=\Delta^{2 k_{r}-1} \partial \alpha=0 .
$$

Thus $(\bigoplus 1) \alpha^{\prime}$ is the fixed set of an involution. However, [2, (24.2)] observes that the real projective space bundle of $(\bigoplus 1) \alpha^{\prime}$ is cobordant to $\left(R P^{2}\right)^{k_{r}}$, which is not a boundary, while the projective space bundle of the fixed set of an involution bounds $[2,(24.1)]$. This is a contradiction.

This completes the induction and the proof of the theorem.

\section{BUNDLES OVER $\left(R P^{1}\right)^{k}$}

The most direct approach to proving the theorem would start by finding the possible Stiefel-Whitney classes for all vector bundles over $\left(R P^{1}\right)^{k}$. Unfortunately, the classes turn out to be surprisingly complicated, and the argument was chosen to bypass this point. It seems desirable to describe the classes.

Proposition. Let $H^{*}\left(\left(R P^{1}\right)^{k} ; Z_{2}\right)=Z_{2}\left[\alpha_{1}, \ldots, \alpha_{k}\right] /\left\langle\alpha_{i}^{2}=0\right\rangle$, where $\alpha_{i}$ is the 1-dimensional class given by projection on the ith factor. There are vector bundles over $\left(R P^{1}\right)^{k}$ having Stiefel-Whitney classes

(1) $1+\alpha_{i}$;

(2) $1+\alpha_{i_{1}} \alpha_{i_{2}}, i_{1}<i_{2}$

(3) $1+\alpha_{i_{1}} \alpha_{i_{2}} \alpha_{i_{3}} \alpha_{i_{4}}, i_{1}<i_{2}<i_{3}<i_{4}$; and

(4) $1+\alpha_{i_{1}} \alpha_{i_{2}} \cdots \alpha_{i_{8}}, \quad i_{1}<i_{2}<\cdots<i_{8}$,

and every bundle over $\left(R P^{1}\right)^{k}$ has Stiefel-Whitney class a product $\Pi\left(1+x_{j}\right)$ for some subset of this set of classes.

Proof. To construct the given classes, let $r=1,2,4$, or 8 and consider the projection $\pi:\left(R P^{1}\right)^{k} \rightarrow\left(R P^{1}\right)^{r}$ corresponding to $\alpha_{i_{1}}, \ldots, \alpha_{i_{r}}$. Compose this with a degree one map to the sphere $S^{r}$ and pull back the $r$-plane bundle over the sphere having $w=1+\sigma_{r}$. 
Being given any vector bundle $\eta$ over $\left(R P^{1}\right)^{k}$, one can choose a unique sum $\sum \rho$ of these bundles for which $w_{i}\left(\sum \rho\right)=w_{i}(\eta)$ for $i \leq 8$. Then, for $\theta=\eta-\sum \rho$,

$$
w(\theta)=1+w_{2^{s}}+\text { higher terms }
$$

with $s>3$.

If one considers the Thom space of $\theta$ with Thom class $U$, one has

$$
S q^{2^{s}} U=w_{2^{s}} U, \quad S q^{i} U=w_{i} U=0 \quad \text { for } 1 \leq i<2^{s} .
$$

From [1], there are secondary cohomology operations $p^{2^{s}-j}, 0<j<2^{s}$, with

$$
\begin{aligned}
w_{2^{s}} U & =S q^{2^{s}} U=\sum S q^{j} p^{2^{s}-j} U=\sum S q^{j}\left(y_{2^{s-j}} U\right) \\
& =\sum y_{2^{s}-j} S q^{j} U=\left(\sum y_{2^{s}-j} w_{j}\right) U=0
\end{aligned}
$$

since Steenrod operations are trivial in $\left(R P^{1}\right)^{k}$. Thus, $w_{2^{s}}(\theta)=0$ and so $w(\theta)=1$.

The most obvious vector bundles over $\left(R P^{1}\right)^{k}$ are the line bundles, giving Stiefel-Whitney classes $1+x$ for every 1-dimensional class $x$. It is immediate that

$$
1+\alpha \beta=(1+\alpha+\beta)(1+\alpha)(1+\beta),
$$

so the classes of 2-plane bundles described above can be given by sums of line bundles.

Surprisingly, the classes of the 4-plane and 8-plane bundles cannot be expressed as sums of line bundles. One has

$$
\left(1+\alpha_{i_{1}}+\cdots+\alpha_{i_{s}}\right)=\left(1+\alpha_{i_{1}}\right) \cdots\left(1+\alpha_{i_{s}}\right) \prod_{u<t}\left(1+\alpha_{i_{u}} \alpha_{i_{t}}\right),
$$

as can readily be seen by induction on $s$, and so the classes of line bundles are all obtained using only the first two types. Thus, sums of line bundles do not give all Steiefel-Whitney classes.

\section{REFERENCES}

1. J. F. Adams, On the non-existence of elements of Hopf invariant one, Ann. of Math. (2) 72 (1960), 20-104.

2. P. E. Conner and E. E. Floyd, Differentiable periodic maps, Springer, Berlin, 1964.

3. Wen-Tsün Wu, Les i-carrés dans une variété grassmannienne, C. R. Acad. Sci. Paris Sér. I Math. 230 (1950), 918-920.

Department of Mathematics, University of Virginia, Mathematics-Astronomy BuILDing, Charlottesville, Virginia 22903-3199 